\title{
A Novel Algorithm For Radar Target Feature Extraction
}

\author{
Shen Limin* \\ The PLA University of Information Engineering Luoyang Campus Luoyang, Henan, 471009, China
}

\begin{abstract}
Due to the aspect sensitivity of High Resolution Range Profile (HRRP), traditional radar target recognition methods usually use average HRRP within some aspect region.Actually,the amplitude fluctuation property of HRRPs also represents some feature information of target.According to the scattering center model, a new feature extraction method using the amplitude fluctuation property of HRRPs is proposed in this paper. The new algorithm mainly extracts the features of the effective distance unit according to the distribution of the target scattering points to improve the recognition efficiency.The experimental results based on the SVM classifier show that the novel feature extraction method can improve recognition performances greatly .
\end{abstract}

\section{Introduction}

Radar target recognition based on High Resolution Range Profile (HRRP) is the mainstream of modern war environment perception and recognition. Radar target recognition based on HRRP avoids complicated motion compensation and can greatly improve the target interception ability to meet the requirements of real-time guidance. HRRP recognition is an important method for the identification of complex military targets and has received extensive attention and in-depth research. Antinoise performance, azimuth sensitivity, and training-set size sensitivity are important indicators of the overall performance of the HRRP classifier ${ }^{[1][2]}$.

Feature extraction is the most critical for radar target recognition. The recognition ability of target recognition system depends greatly on the selection of feature vectors and the accuracy of feature extraction. Most of the radar observations are in the optical scattering region, the target can be equivalent to a set of multiple isolated scattering centers. The scattering center contains features such as position, amplitude, and geometry type. Extracting these features accurately has important application value in the fields of automatic target recognition and self-focusing. At present, the feature extraction method based on HRRP is mainly divided into non-parametric methods and parameterized methods ${ }^{[3-5]}$.

The typical representation of non-parametric methods is the direct use of HRRP. Li and Yang elaborated the feasibility of directly using HRRP as feature vectors, and proposed a recognition method based on matching degrees The typical representation of the parametric method is the Relax algorithm based on the simple scattering point model, this algorithm is based on the Clean algorithm. Based on the Relax algorithm, this paper presents an improved feature extraction method, which uses the position and intensity of the scattering center as the target feature to extract the geometric parameters of the target structure.

\section{Data model of Echo signal}

The simple scattering point model theory is that when the radar work in optical region, the target can be composed of several discrete scattering points, and the radar target echo is the vector sum of the sub-echo formed by the backscattering of the emission point by each scattering point ${ }^{[6-8]}$. Relax algorithm is a nonlinear least squares relaxation algorithm for solving simple scattering point model parameters.In this paper, by using the average distance of the Relax algorithm for radar echo as feature extraction. Formula (1) is the simple scattering point model of radar target withe noise ${ }^{[9]}$.

$$
y_{n}=\sum_{k=1}^{K} \alpha_{k} e^{j 2 \pi f_{k} n}+e_{n}
$$

$k$ represents the number of scattered points of radar exposure at a specific target azimuth; $\alpha_{k}$ is the complex envelope of the kth scattering point echo, which is a function of time and represents the radar target intensity; $f_{k}$ is the kth sine wave frequency, which means the distance to the position information. Assuming $e_{n}$ is white noise, in the Relax algorithm, the noise relaxation is the self-regression process. as follows:

$$
e_{n}=\sum_{m=1}^{M} a_{m} e_{n-m}+w_{n} n=0,1, \cdots N-1
$$

$w_{n}$ represents a zero-mean complex Gaussian white noise process

\footnotetext{
*Corresponding author: shenlimin1996@163.com
} 


$$
w\left(f_{k}\right)=\left[1, e^{j 2 \pi f_{k}}, e^{j 2\left(2 \pi f_{k}\right)}, \cdots, e^{j(N-1) 2 \pi f_{k}}\right]^{T}
$$

Rewrite the above model in matrix form

$$
Y=A \alpha+e_{n}
$$

The problem becomes to solve the parameters based on the above model and radar data

$$
\left\{f_{k}, a_{k}\right\}=\arg \min \underset{\left\{f_{k}, a_{k}\right\}}{\arg \min }\|Y-A \alpha\|
$$

\section{Radar target feature extraction}

Radar HRRP recognition is mainly divided three steps: Gaining HRRP; Feature Extraction for Radar target; Classifier, Flow chart shown in Figure 1.

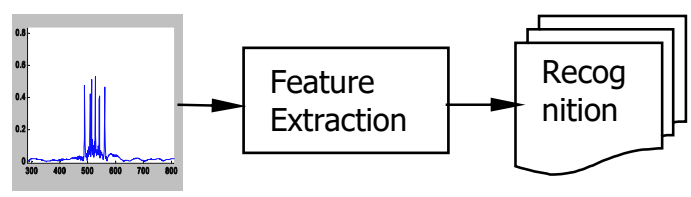

Fig. 1. Flow chart of Radar HRRP Recognition

\subsection{Pre-processing of HRRP}

Pre-processing method of HRRP is introduced in order to strengthen useful information and restore the degenerated phenomenon which was caused by the other factors. The following pre-processing method was used in this paper.

\subsubsection{Power Transformation}

HRRP is obtained through the absolute duplicate value of HRRP data, regarding the $\Gamma$ positive solidly vector process, every variable can obey the distribution approximately, correspondingly, $\Gamma$ distribution can transform the kind of Gaussian distribution through power transformation. The power transformation is defined as:

$$
y=x^{v}, 0<v<1
$$

Where $v$ are coefficients of power transformation.

\subsubsection{Normalization}

The centre of goal scattering position and the relative scope is emphasized of HRRP classification, normalizations each goal's HRRP according to the biggest peak value is not only eliminate dependence of he goal but also simplify hunting zone of the parameters $\sigma$. If specify it has $\mathrm{n}$ samples in pattern classes, Normalized processing as following equation (7).

$$
x^{-(i)}=\frac{x_{j}^{(i)}}{\left\|x_{i}^{(i)}\right\|}\{i=1,2, \cdots c ; j=1,2, \cdots N\}
$$

\subsection{A Novel Algorithm Based on Relax}

Improved algorithm focuses on the selection of effective distance units, it can be considered from two aspects: firstly there are almost no scattering point echoes on some distance units, or there are only some very weak clutter and noise; Secondly, there are many scattering points in some distance unit distribution, but there is no obvious effect. an improved algorithm is proposed to solve the above problems, Figure 2 is a flowchart of an improved algorithm. Assuming the parameters $\left\{a_{k}, f_{k}\right\}$ have been estimated, Signal $\sum_{i=1, i \neq k}^{K} a_{i} w\left(f_{i}\right)$ reconstructed from a simple scatter point model has a residual signal .

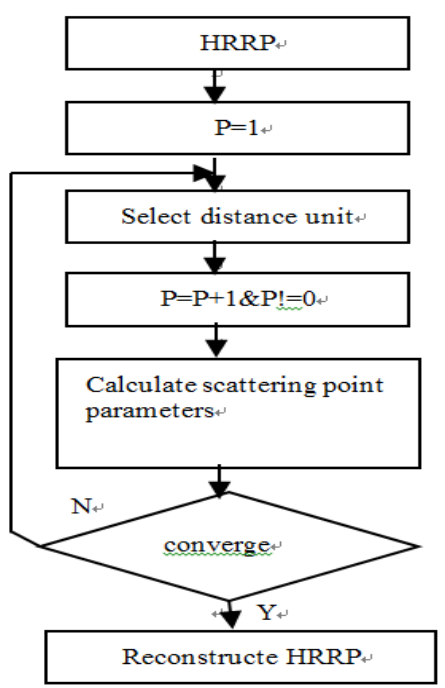

Fig.2 The flow chart of the improved algorithm

$$
\begin{aligned}
& y_{k}=Y-\sum_{i=1, i \neq k}^{K} a_{i} w\left(f_{i}\right)=Y-w a+w\left(f_{k}\right) a_{k} \\
& a_{k}=\frac{w^{T}\left(f_{k}\right) y_{k}}{N}=\frac{D F T\left(y_{k}\right)}{N} \\
& f_{k}=\arg \max _{f_{k}} \arg \left\|\operatorname{DFT}\left(y_{k}\right)\right\|^{2}
\end{aligned}
$$

\section{Simulation and analysis}

Support Vector Machines(SVM) are used as classifiers to perform training classification to verify the effectiveness of the proposed algorithm. HRRP of the radar target V-22 with pitch angle and horizontal azimuth spacing is simulated. The simulation parameters are carrier frequency $10 \mathrm{GHz}$,bandwidth $150 \mathrm{MHz}$ (corresponding distance resolution $1 \mathrm{~m}$ ), sampling points 200 , signal to noise ratio $10 \mathrm{Db}, \mathrm{A}$ novel Relax algorithm convergence threshold set to 10-3. The influence of the number of scattered points and the data processing speed are verified.

\subsection{Influence of Number of Scattering Points on Reconstructed HRRP}

The experimental results are shown in Fig.3, as follows (a), (b), (c), and (d) are reconstructed from the scattered points of $18,15,8$ and 6 respectively. 


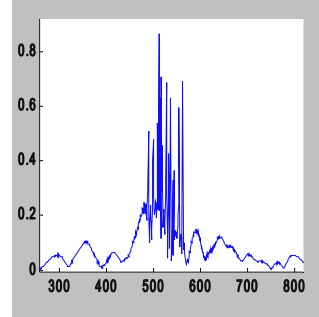

(a)

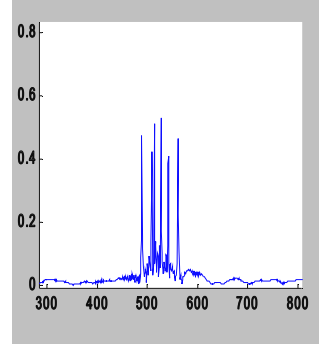

(c)

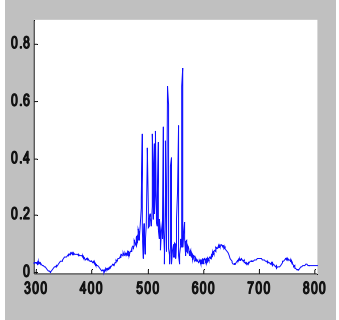

(b)

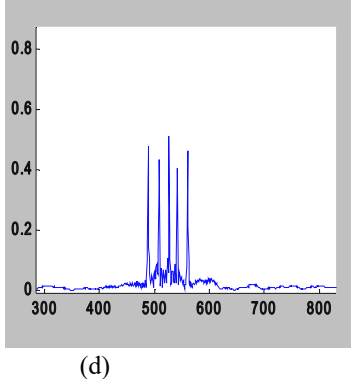

Fig.3 The restructuring HRRP of choosing different scattering spots

It can be seen from the Fig. 3 that the target scattering point parameters extracted by the RELAX algorithm can reflect the scattering point distribution of the original range profile. From the simulation diagram, the more the scattering points are extracted from the target echo, the closer the reconstruction range profile is to the original range profile, and more beneficial to the design of the classifier. It can be seen from Fig. 3 (c) and (d) that when the number of scattered points is less than 10, the complete distance image cannot be constructed. Fig. 3(a), (b) When the number of scattering points are greater than 15, the reconstructed range profile has a small difference from the original range profile.

\subsection{Recognition rate}

Table 1 shows the simulation results from correct recognition rate. It can be seen from Table 1 that the improved algorithm has considerable advantages.

Table 1. Compared the performance with Relax and improved Relax.

\begin{tabular}{cccccc}
\hline & scattering points & 18 & 15 & 8 & 6 \\
\hline $\begin{array}{c}\text { Relax } \\
\text { algorithm } \\
\text { improved } \\
\text { Relax }\end{array}$ & $\begin{array}{c}\text { Correct recognition } \\
\text { rate }\end{array}$ & 0.97 & 0.96 & 0.96 & 0.93 \\
\hline
\end{tabular}

\section{Conclusions}

This paper first analyzes the data model of the target echo signal. Based on this model, the Relax algorithm is deeply analyzed. The characteristics of the radar target HRRP are initialized. With the improved Relax algorithm, the characteristics of the scattering point parameters are extracted from the measured data of the target V-22 radar echo. The simulation system based on MATLAB is established and the distance profile is reconstructed. Finally, the simulation results show that the improved algorithm is effective in selecting the number of scattered points and data processing speed.

\section{References}

1. $\mathrm{Li} \mathrm{H}$, Yang $\mathrm{S}$. Using range profiles as feature vectors to identify aerospace object[C]. IEEE Trans. on AP. 41 (3):261-280 (1993).

2. Huang Deshuang, Technology of Intelligent Signal Processing Based On High Resolution Radar[M]. Beijing: Publishing Of Machinery Industry(2001).

3. H-J.Li,Y.D,Wang,L-H.Wang,Matchingscore properties between range profile of high resolution radar targets, IEEE Antennas and Propagation Magazine[J]. 44(4): 444-452 (1996).

4. Xing $\mathrm{M}, \mathrm{Wu} \mathrm{R}$, Lan J, et al.Migration Through Resolution Cell Compensation in ISAR Imaging[J].IEEE GRS.Letters , 1(2) : 360400,(2004).

5. BAO zheng,Xing Mengdao,Wang tong. Radar Imaging Technique[M] Beijing: Publishing Of Electronics Industry,( 2015).

6. Pan mian. Study of Radar Target Recognition using Range Profiles Based on Time Frequency Features and Kernel Methods [D],ChengDu: University of Electronic Science and Technology of China.(2017)

7. ZHANG xuefeng. Study on Radar HRRP Target Recognition[D]. XI AN : Xidian University.(2015)

8. ZHU jiehao Research on Radar Target High Resolu- tion Range Profile Simulation and Recognition [D]. NANJING: Nanjing University of Aeronautics.(2012)

9. ZHANG zhongming. Research on Radar Target High Resolution Range Profile Simulation and Recognition [D]CHANGSHA: National Defense University,(2014) 